and Laboratory Report, June I886, of which I inclose a marked copy. It will be observed that while Ulrich accepts the identity of the New Zealand alloy with oktibehite, Skey's analysis shows that its formula is $2 \mathrm{Ni}+\mathrm{Fe}$, while that of the latter mineral is $\mathrm{Ni}+\mathrm{Fe}$.

N.Z. Geological Survey Office, Wellington, N.Z., February 9

\section{AËRIAL VORTICES AND REVOLVING SPHERES}

A

STRIKING series of experiments on aërial vortices and revolving spheres has lately been made by $M$. Ch. Weyher, one of the directors of the important establishment for mechanical constructions at Pantin. An account of these experiments, with illustrations, appeared in a recent number of La Nature (February 26). As the results obtained by $M$. Weyher are very interesting, we reproduce the more important figures, and translate the descriptions given by our French contemporary.

Fig. I.-Aërial Vortices.-A glass cylinder of about $0.40 \mathrm{~m}$. in diameter by $0.70 \mathrm{~m}$. in height, has an upper cover, pierced by a hole through which passes the shaft of the drum, the latter being formed of one or two paddles of cardboard put cross-wise upon the vertical shaft.

The cylinder contains some sawdust, or, better still, some oatmeal. If the oatmeal is put at first so as to form

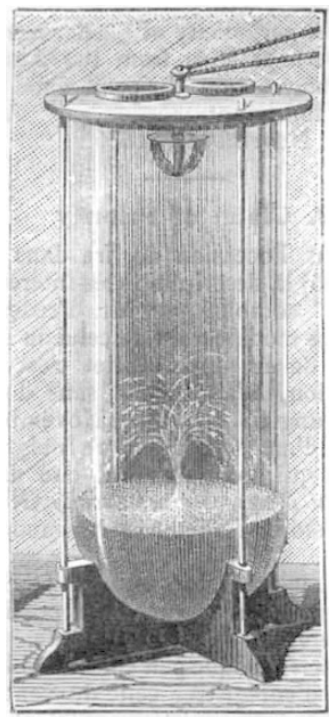

Fig. I.

a cone or mound, and if the drum is turned round, a little waterspout can be seen forming at the top of the mound. Gradually the mass of oatmeal sinks into a hemisphere.

The matter runs without ceasing into spirals from the circumference to the centre ; there it forms at first the lower cone, and then the upper reversed cone, in which the particles of oatmeal describe spirals, going from the centre to the circumference.

The whole system describes a primary general sphere, more or less distorted, the centre of which (where the two cones meet) is more or less deranged by the earth's gravity. If this is looked at from above, a hollow funnel is seen upon the axis : it is there that the air is most rarefied by rotation, and it is there that the finest particles come.

Substituting for oatmeal in the apparatus small light balloons inflated with air, the general movement can be followed. When the balloons are on the exterior circum- ferences, they fall in slow spirals; when they reach the circumferences nearer the axis of rotation, they rise rapidly upon a helix at a much more extended pace. In short, the experiment shows that, given a mass of air, if a

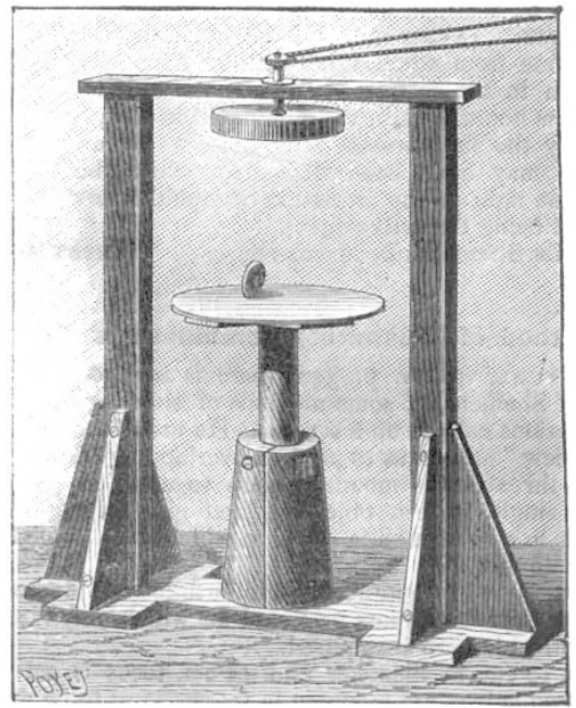

FIG. 2.

movement of rotation round a vertical axis is imparted to it this air falls constantly by the exterior circumferences, and rises by the interior circumferences, and the whole volume passes unceasingly through the centre of

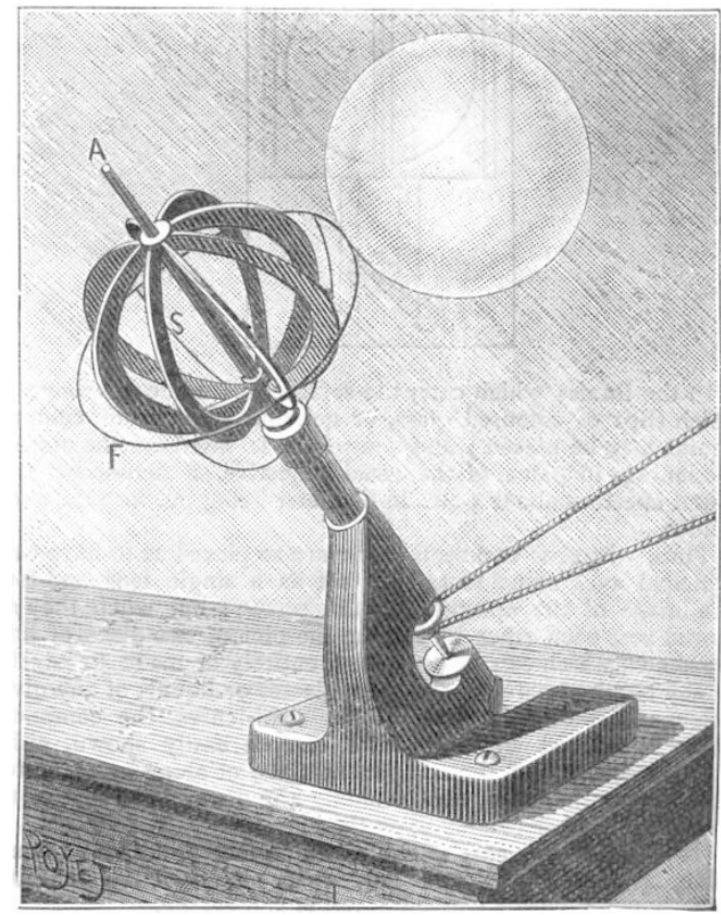

F1G. 3 .

the vorte: drawing intn it s movement the substances or particles therein im nersed.

Fig. 2. - A plate of glass or any other material is placed below a drum with paddles; when this drum is put in 
motion, we immediately place upon the plate a disk or a coin, to which the fingers give a first movement of rotation round one of its diameters.

The hand being quickly drawn back, the aërial vortex continues to make the coin turn round like a top, and absolutely keeps it captive in its radius of action. The coin, while turning upon one of its diameters, makes a sphere, and a later experiment will show that a revolving sphere constitutes a centre of attraction.

Fig. 3.-Equilibrium of Revolving Spheres.-A free sphere keeps itself in equilibrium, and turns round another sphere, to which a rapid movement of rotation is imparted.

The apparatus consists of a pin, A, which is able to turn in a support, and has a pulley, made to receive a transmitted movement. Upon the pin A is placed a sphere, $s$, composed of from eight to ten flat circular pieces (these may be either flat disks, or disks cut into a crescent shape; it does not matter which). The pin may be at any angle whatever to the horizon; in this experiment it is inclined at $45^{\circ}$, but it may be horizontal

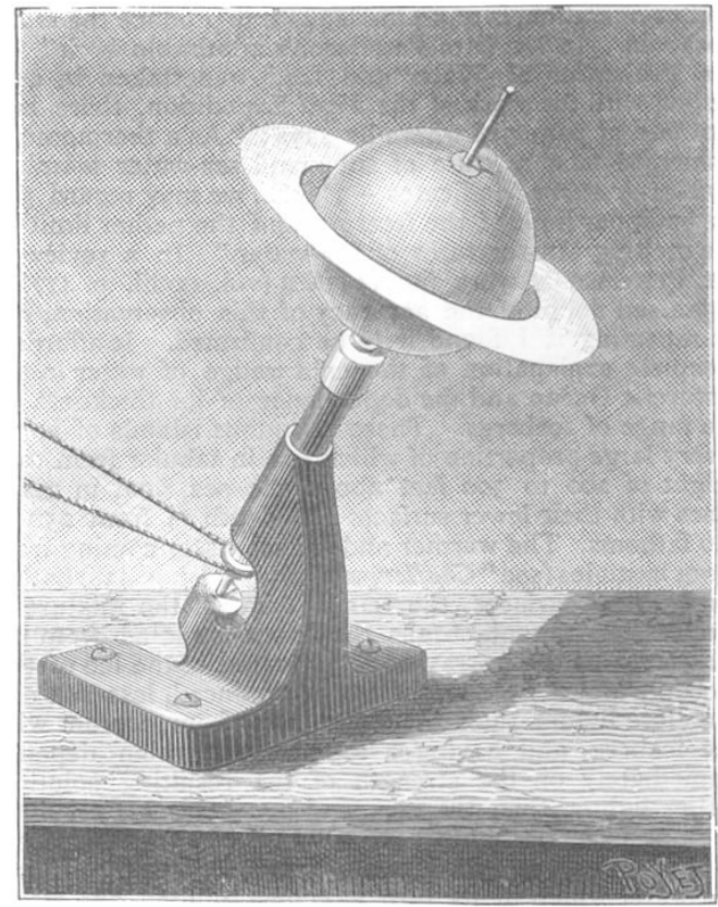

FIG. 4 .

or vertical. The angle of $45^{\circ}$ was chosen because it seemed to be imost difficult for the experiment, which would therefore be the more conclusive. When the sphere $S$ is turned round rapidly, you feel on the hand a strong blast which escapes all around from its equator. Bits of paper which are placed near it are thrown far away. Nevertheless, if a balloon is put near this blast, it is quickly attracted towards the revolving sphere, and describes orbits round it in the plane of the equator. As the experiment took place in a room, where there were obstacles producing eddies, and as also gravity has too much effect by reason of the proximity of the ground, it is very difficult to obtain a regular movement. The balloon comes easily in contact with the revolving sphere, and is then driven away by the collision too far to be caught again. A very simple contrivance consists in placing round the sphere $\mathrm{S}$ a wire guard or circle of iron, F, I millimetre in diameter, attached to the support by three similar wires.

The balloon will then keap on turning round the motive sphere, even ceasing to touch the guard in the lower part under the action of gravity. The.experiment can be made in different ways, and the guard may even be suppressed, but these variations teach us nothing new.

In studying the vortex movements which the sphere imparts in the medium in which it is plunged, we easily calculate the ratio of the attraction which it exercises on the balloon.

Fig. 4.-The guard of the revolving sphere is taken away, and we place parallel to its equator a circle of paper with an interior diameter greater than the exterior diameter of the sphere ; the circle is caught into the movement of rotation, and maintains itself strongly in the plane of the equator.

\section{$O N O L D H A M I A$}

$\mathrm{THE}$ organic origin of Oldhamia has often been disputed Originally described by Edward Forbes from specimens found at Bray Head, near Dublin, in rocks of the Cambrian formation, it has been found in a few other localities in Ireland, in rocks of a similar formation. In the dispute the weight of the evidence has seemed to be in favour of the views of Forbes, Jukes, Harkness, and Kinahan, that this lowly form is a fossil probably belonging to the Polyzoa, or to the Sertularian Polyps. So the matter has stood for a long time. A recent paper by Prof. W. J. Sollas, published in the Proceedings of the Royal Dublin Society for January last, once again opens the discussion. In the hope of throwing light on this problematical structure, thin slices for microscopic examination were cut, both parallel and transverse to its planes of cleavage. When these were placed under the microscope, all trace of the Oldhamia structure appeared to have vanished. An examination with the unaided eye showed, however, that it was still there, presenting itself as narrow, undulating, and branching bands of a lighter colour than the surrounding matrix. Its appearance, moreover, varied in an extraordinary manner according to the direction in which it was viewed. Looked at obliquely in a strong light, the thread-like bands are brilliantly illuminated, and appear fintly coloured with spectral tints; looked at directly, the bands become fainter, and are less clearly distinguishable from the matrix. In certain positions the slice taken at right angles to the bedding has an appearance somewhat suggestive of shot-silk, and, from the planes of cleavage, markings which remotely resemble in form the dendritic markings of Sutton stones extend into the surrounding matrix.

These appearances suggest the presence of some mineral possessing high reflection or refraction arranged in more or less parallel planes. Mr. Teall, in the same paper, gives full details of the mineral characters of the rock. Aided by these, Dr. Sollas finds that the lighter-coloured bands, which correspond to the Oldhamia markings, owe their distinction from the surrounding matrix to the presence of an excess of sericite scales; and that the curious shot-silk appearances are produced by the local deflections of these scales from parallelism with the cleavage planes into directions tangential to curves, which are probably transverse sections of those long ridges which, when seen on the exposed surface of a cleavage plane, are recognised as the usual form of Oldhamia. It would appear possible that these ridges are wrinklings of the cleavage planes produced during the shearing which led to their formation. These observations were made on the form known as O. radiata, and in some supplementary remarks Dr. Sollas adds that when Oldhamia is present it shows itself on the surface of the laminæ as rounded discontinuous ridges, which are without definite boundaries, and have the appearance of fine wrinkles. When the phyllode is fractured obliquely to the cleavage laminæ, the Oldhamia markings are found to extend through the rock as fine ridges or wrinkles 